\title{
TRIPLE AND MULTIPLE SYSTEMS, THEIR GEOMETRIC CONFIGURATIONS AND GROUPS*
}

\author{
BY \\ ARNOLD EMCH \\ I. INTRODUCTION
}

The fact that every finite group may be represented as a substitution group of a certain degree $m$ makes it possible to construct in every case certain configurations in a projective space of $m-1$ or lower dimensions whose points correspond uniquely to the substitutions of the group. Such configurations are representatives of the groups and are characterised by geometric properties which reflect the properties of the group. Such representations in certain particular cases are numerous and have been known for a long time. From this standpoint I have considered the symmetric group of degree $m$ on a hyperquadric in $S_{m-1} . \dagger$ It is thus obvious that any finite group may be represented by a certain configuration (with characteristic geometric properties) on a hyperquadric. Whether such a representation would in every case be mathematically profitable is an open question. While there is thus indicated a general method of approach to the geometrical problem of finite group theory, there may be developed special methods of representation of finite groups in connection with particular problems connected with group theory, which seem to be of sufficient interest and importance to deserve attention. This is the case with the theory of triple and multiple systems, where geometric considerations are found in the literature only in a few instances. $\ddagger$ It was Kirkman $\S$ in 1844 , and Steiner in 1853 who first stated the problem of triple systems and certain multiple systems in its most elementary aspect as it was revealed by the study of the configuration of

* Presented to the Society, September 8, 1926, and April 6, 1928; received by the editors March 31, 1928.

† Some geometric applications of symmetric substitution groups, American Journal of Mathematics, vol. 45 (1923), pp. 192-207. 477-508.

$\ddagger$ L. Heffter, Ueber das Problem der Nachbargebiete, Mathematische Annalen, vol. 38 (1891), pp.

De Vries, Zur Theorie der Tripel Systeme, Rendiconti del Circolo Matematico di Palermo, vol. 8 (1894), pp. 222-226. Ueber polyedrale Configurationen, Mathematische Annalen, vol. 34 (1889) pp. 237-246.

\$On a problem in combinations, The Cambridge and Dublin Mathematical Journal, vol. 2 (1847), pp. 191-204. 
the 28 double tangents of the quartic.* A detailed account of this connection is given by Noether in an important paper $\dagger$ which appeared in 1879 . Netto pays attention to the algebraic properties of triple systems and the closely associated triple equations and their groups. Since that time triple systems have been studied by a number of investigators mostly with reference to existence proofs, formation and group properties of such systems. $\neq$

That a vast number of similar systems, for example triple systems with double couples, quadruple systems containing every triple once, in general n-tuple systems of various types, may be formed becomes evident without much effort. There seems to be little that has been done along this line of generalization. Inasmuch as with every well defined system of this sort there is associated a finite group which dominates it and as the number of such systems is unlimited, there exists a large class of finite groups which bear conversely the characteristics of these systems. Each triple or multiple system and couples of such may be represented by a certain spatial configuration to which is attached a related finite group. It is the purpose of this paper to show by a few simple examples how such configurations and their groups may be constructed.

\section{Triple systems}

1. Simple triple systems. As systems of this sort, their description and some of their properties, at least for lower orders, are fairly well known I shall not enter into a detailed discussion of them, and shall present merely a few additional circumstances which seem to be worthy of notice.

It is well known that triple systems $\Delta_{m}^{8}$ on $m$ elements exist only, and in every case, for numbers of the form $m=6 m+1$, and $m=6 m+3$. Instead 181-182.

* Combinatorische Aufgabe, Journal für reine und angewandte Mathematik, vol. 45 (1853), pp.

†Ueber die Gleichungen achten Grades und ihr Auftreten in der Theorie der Curven vierter Ordnung, Mathematische Annalen, vol. 15 (1879), pp. 89-110.

$\ddagger$ E. Netto, $Z$ ur Theorie der Tripelsysteme, Mathematische Annalen, vol. 42 (1893), pp. 141-152.

E. H. Moore, Concerning triple systems, Mathematische Annalen, vol. 43 (1893), pp. 271-285.

F. N. Cole, The triad systems of thirteen letters, these Transactions, vol. 14 (1913), pp. 1-5.

H. S. White, Triple systems as transformations, and their paths among triads, these Transactions, vol. 14 (1913), pp. 6-13.

Louise D. Cummings, On a method of comparison for triple systems, these Transactions, vol. 15 (1914), pp. 311-327. 13-19.

H. S. White, The multitude of triad systems on 31 letters, these Transactions, vol. 16 (1915), pp.

S. Bays, Sur les systèmes cycliques de Steiner, Annales Scientifiques de l'Ecole Normale Supérieure, (3), vol. 4 (1923), pp. 55-96.

In these papers may also be found additional references. 
of using the medium of hyperquadric or other forms of possible graphic representation we may make use of the simple device of representing the $m$ elements by $m$ generic points in ordinary space $S_{3}$. Then to every triple system corresponds a certain open polyhedral configuration of triangles on these $m$ points. To two triple systems $\Delta_{m}{ }^{3}$ without a common triple corresponds a closed polyhedral configuration, whose mapping upon simple closed surfaces will be explained subsequently in case of $m=7$ and $m=9$.

$\Delta_{7}{ }^{3}$. For certain purposes (determination of the order of the group, etc.) it is desirable, although not necessary, to have some sort of a generalized matrix from which we may write down a triple system in a very simple and effective manner. The terms for $m=7$, for seven elements $1,2, \cdots, 7$, may be written in any order $a, b, \cdots, g$ in the form

$$
\left\|\begin{array}{lll}
a & b & c \\
& d & \\
e & f & g
\end{array}\right\|
$$

from which the 7 triples are immediately obtained as (1) the first line, (2) the second column, (3) and (4), the two diagonals, (5) and (6), the first and third column each together with $f$, (7) the triangle $b e g$; i.e., $a b c, b d f$, $a d g$, $c d e, a$ ef, $c g f, b e g$. Each such form or "matrix" may be identified by a substitution

$$
\left(\begin{array}{lllllll}
1 & 2 & 3 & 4 & 5 & 6 & 7 \\
a & b & c & d & e & f & g
\end{array}\right) .
$$

It is easy to write down the 24 "matrices" with the central letter $d$ unchanged which produce the same triple system. The 24 corresponding substitutions form a subgroup of order 24 , and as the central $d$ may be replaced by any of the remaining letters we get a group of $24 \cdot 7=168$ substitutions, the simple $G_{168}$ of this order. As the structure of this group is well known, details of its construction by the foregoing scheme are not necessary.

There are altogether $7 !: 168=30$ distinct $\Delta_{7}{ }^{3}$ 's and any of the 35 triples is always common to 6 distinct $\Delta_{7}{ }^{3}$ 's. But it is not possible to form 5 distinct $\Delta_{7}{ }^{3}$ 's which together contain all of the 35 triples, as has already been established by Cayley.*

$\Delta_{\vartheta}{ }^{3}$. For the generating "matrix" we may use the square arrangement

$$
\left\|\begin{array}{lll}
a & b & c \\
d & e & f \\
g & h & i
\end{array}\right\| .
$$

* Papers, vol. 1, pp. 481-484. 
The 12 triples obtained from the three rows, the three columns and the 6 terms of the ordinary determinant expansion form a triple system $a b c, d e f, g h i, a d g, b e h, c f i, a e i, a f h, b d i, c d h, b f g, c e g$, on 9 letters or numbers, in which $a, b, \cdots, i$ denote the 9 numbers $1,2, \cdots, 9$ in any order. Thus the particular arrangement of the "matrix" is equivalent to the substitution

$$
\left(\begin{array}{lllllllll}
1 & 2 & 3 & 4 & 5 & 6 & 7 & 8 & 9 \\
a & b & c & d & e & f & g & h & i
\end{array}\right) .
$$

To determine the group which leaves the triple system invariant we notice first that the $\Delta_{9}{ }^{3}$ as a whole does not change when any two rows, or any two columns are interchanged. This gives a subgroup of 36 substitutions which leave $\Delta_{\ominus}{ }^{3}$ invariant.

But there are 11 other distinct matrices and distinct from any attached to the $G_{36}$, which also leave $\Delta_{9}{ }^{3}$ invariant, namely (including the first)

$$
\begin{array}{l|lll}
\left\|\begin{array}{lll}
a & b & c \\
d & e & f \\
g & h & i
\end{array}\right\| & \left\|\begin{array}{lll}
a & d & g \\
b & e & h \\
c & f & i
\end{array}\right\| & \left\|\begin{array}{lll}
a & e & i \\
b & f & g \\
c & d & h
\end{array}\right\| & \left\|\begin{array}{lll}
a & f & h \\
b & d & i \\
c & e & g
\end{array}\right\| \\
\left\|\begin{array}{lll}
a & b & c \\
e & f & d \\
i & g & h
\end{array}\right\| & \left\|\begin{array}{lll}
a & d & g \\
e & h & b \\
i & c & f
\end{array}\right\| & \left\|\begin{array}{lll}
a & e & i \\
f & g & b \\
h & c & d
\end{array}\right\| & \left\|\begin{array}{lll}
a & f & h \\
d & i & b \\
g & c & e
\end{array}\right\| \\
\left\|\begin{array}{lll}
a & b & c \\
f & d & e \\
h & i & g
\end{array}\right\| & \left\|\begin{array}{lll}
a & d & g \\
h & b & e \\
f & i & c
\end{array}\right\| & \left\|\begin{array}{lll}
a & e & i \\
g & b & f \\
d & h & c
\end{array}\right\| & \left\|\begin{array}{lll}
a & f & h \\
i & b & d \\
e & g & c
\end{array}\right\| .
\end{array}
$$

With each of these are connected as above 36 substitutions which leave $\Delta_{9}{ }^{3}$ invariant. These together form all the substitutions which leave the given $\Delta_{9}^{8}$ invariant, and they consequently form a $G_{432}$ of order $36 \cdot 12=432$, which is known as the group of the triple equation of degree 9. There are, of course, several ways of generating this group. For instance if we set

$$
\begin{aligned}
& S_{1}=123 \cdot 456 \cdot 789, \\
& S_{2}=147 \cdot 258 \cdot 369, \\
& S_{3}=159 \cdot 267 \cdot 348, \\
& S_{4}=168 \cdot 249 \cdot 357,
\end{aligned}
$$

then $G_{9}\left(S_{1}, S_{2}, S_{3}, S_{4}\right)$ is a non-cyclic abelian group of order 9 and the $G_{432}$ is the holomorph of $G_{9}$. 
Each triple is common to 120 distinct triple systems.

If we consider for example the triple 123 fixed, then the remaining numbers form 6 ! permutations $a b c d e f$, each giving the second and third row in the "matrix," namely $a b c$ and $d e f$. But as these may be interchanged and a cycle of order 3 applied to each without changing $\Delta_{9}{ }^{3}$, there are evidently only $6 !: 6=120$ permutations which with 123 give distinct $\Delta_{9}{ }^{3}$ 's. Now it is clear that two triples with a common couple, for example 123 and 124, cannot belong to the same $\Delta_{9}{ }^{3}$. Moreover every couple must occur in every $\Delta_{9}{ }^{3}$ just once. Hence all $\Delta_{9}{ }^{3}$ 's can be classified in various ways into 7 classes of 120 triple systems, each class being characterised by a common triple. Thus $123,124,125,126,127,128,129$ are each common to $120 \Delta_{9}{ }^{3}$ 's. Thus there are altogether $7 \cdot 120=840$ distinct triple systems on 9 elements. This checks up with the fact that $9 !: 432=840$.

Now in case of 9 the remarkable circumstance presents itself that all 84 triples may be distributed completely and each only once in 7 triple systems. This may be called a complete set of triple systems.

That this is not always possible is clear from the case of $m=7$. Such a complete set is for example obtained from the 7 matrices

$$
\left\|\begin{array}{lll}
1 & 2 & 3 \\
4 & 5 & 6 \\
7 & 8 & 9
\end{array}\right\| \quad\left\|\begin{array}{lll}
1 & 2 & 4 \\
3 & 5 & 9 \\
7 & 6 & 8
\end{array}\right\|\left\|\begin{array}{lll}
1 & 2 & 5 \\
3 & 7 & 8 \\
9 & 4 & 6
\end{array}\right\|\left|\begin{array}{lll}
1 & 2 & 6 \\
3 & 4 & 7 \\
8 & 5 & 9
\end{array}\|\| \begin{array}{lll}
1 & 2 & 7 \\
3 & 6 & 8 \\
4 & 9 & 5
\end{array}\left\||| \begin{array}{lll}
1 & 2 & 8 \\
4 & 3 & 9 \\
5 & 7 & 6
\end{array}\right\|\left\|\begin{array}{lll}
1 & 2 & 9 \\
7 & 3 & 6 \\
8 & 4 & 5
\end{array}\right\| .\right.
$$

As there are 840 distinct $\Delta_{9}{ }^{3}$ 's, and from the fact that there exists at least one complete set, it is easily seen that a great number of such complete sets may be formed, and that each $\Delta_{9}{ }^{3}$ may belong to more than one complete set. So far I have not been able to establish necessary and sufficient conditions on the number $m$ for which complete sets of triple systems are possible. The original complete set of $\Delta_{9}^{3}$ 's were found by trial.

2. Triple systems with double couples. $n$ elements form $\frac{1}{6} n(n-1)(n-2)$ triples and $\frac{1}{2} n(n-1)$ couples. Let $\Delta$ be a triple system containing every couple twice and let $x$ be the number of triples of the system. As every triple contains 3 couples,

$$
3 x=\frac{2 \cdot n(n-1)}{2}=n(n-1),
$$

and $x=\frac{1}{3} n(n-1)$. Hence $n$ must have either the form $n=3 m$, or $n=3 m+1$, and

$$
x=m(3 m-1), \quad \text { or } \quad x=(3 m+1) m .
$$

For $m=1$ we get in the first case $m=3, x=2$, a triangle 123 counted twice; in the second case $m=4, x=4$, or the tetrahedron $123,124,134,234$. 
The groups which leave this dihedron and tetrahedron invariant are of course the dihedral group on three letters, or the symmetric group, and the tetrahedral group.

For $m=2$, we have for two cases $n=6, x=10$ and $n=7, x=14$.

In general, for $m=2 \mu$,

or

$$
n=6 \mu, \quad x=2 \mu(6 \mu-1),
$$

For $m=2 \mu+1$,

$$
n=6 \mu+1, \quad x=2 \mu(6 \mu+1) .
$$

$$
n=6 \mu+3, \quad x=2(2 \mu+1)(3 \mu+1),
$$

or

$$
n=6(\mu+1), x=2(2 \mu+1)(3 \mu+2) \text {. }
$$

In case of an even $n$, i.e., $n=6 \mu$, or $n=6(\mu+1)$ we have triple systems with double couples which form closed surfaces. In case of odd numbers, $n=6 \mu+1$, $n=6 \mu+3$, we have the same number as in triple systems with single couples, but twice the number of triples. Hence if we take two such triple systems with no common triple we have a triple system with double couples.

Thus when $\mu=1, n=7, x=14$. When $\mu=1$, in the other case, $n=6+3=9$, $x=24$.

3. Triple system of order 7 with double couples. Such a system may be constructed by taking two ordinary triple systems of order 7 with single couples without a common triple. For example

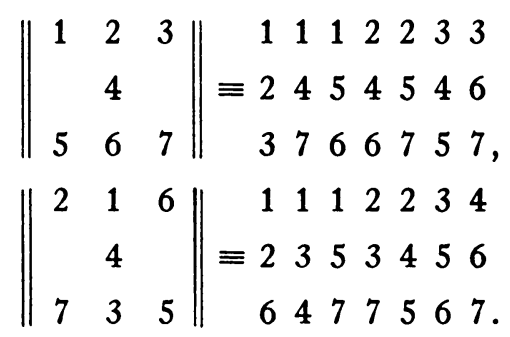

Each system is invariant in a simple $G_{168}$. There are 7 substitutions which permute the two systems, namely

$$
\begin{aligned}
& S_{1}=(27)(35)(46) \text { leaves (1), } \\
& S_{2}=(15)(34)(67) \quad \text { " (2), } \\
& S_{3}=(16)(25)(47) \quad \text { " (3), } \\
& S_{4}=(12)(36)(57) \quad \text { " (4), } \\
& S_{5}=(14)(26)(37) \quad \text { " (5), } \\
& S_{6}=(17)(45)(23) \quad \text { " (6), } \\
& S_{7}=(13)(24)(56) \quad \text { “ (7) }
\end{aligned}
$$


in each system invariant. Then the product of each of these is a cyclic substitution of order 7 and all these products are the powers of one of these. For example

$$
\begin{aligned}
& S_{1} S_{2}=\left(\begin{array}{lllllll}
1 & 5 & 4 & 7 & 2 & 6 & 3
\end{array}\right)=T, \\
& S_{3} S_{5}=\left(\begin{array}{lllllll}
1 & 2 & 5 & 6 & 4 & 3 & 7
\end{array}\right)=T^{4} .
\end{aligned}
$$

$T$ and its powers transform the two triple systems into themselves. Hence this is a subgroup

$$
G_{14}=1, S_{1}, \cdots, S_{7}, T, T^{2}, \cdots, T^{6}
$$

which leaves the triple system $\Delta \tau^{2}$ with double couples invariant.

If we let $1,2, \cdots, 7$ represent generic points in a space of three dimensions, so that each triple is represented by a triangle, then the two systems form a closed surface such that at each of the 21 couples there are two adjacent triangles. This surface can be developed in the manner indicated by the Fig. $1_{\mathrm{a}}$ and then, by uniting the edges $\overline{1} \overline{7}$, Fig. $1_{\mathrm{b}}$, formed into a cyclinder. By deformation the upper rim can be shifted in a clockwise direction, so that corresponding equal numbers are in generatrices of the deformed cylinder.

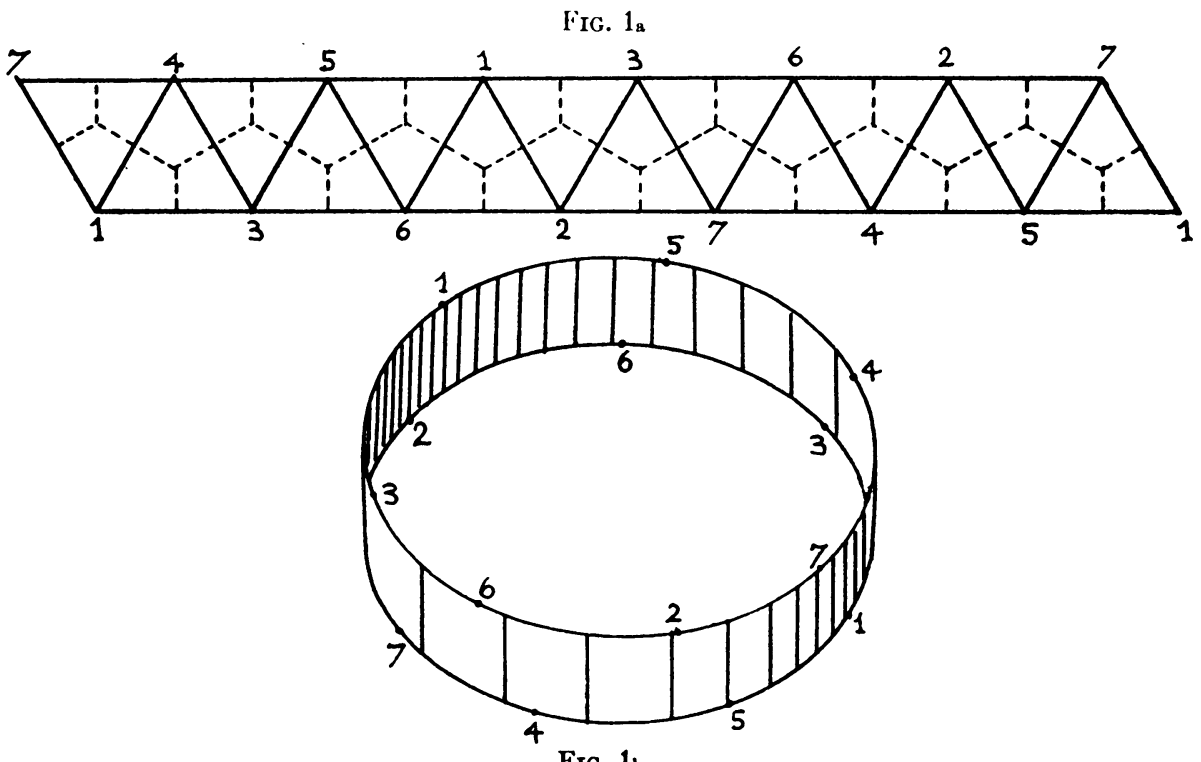

Then the cylinder can be deformed into a torus, by uniting equal numbers. By joining the centers of the triangles as indicated in the figure we obtain 7 hexagons on the torus with the seven points as centers. For example, the centers of the triangles $134,147,175,156,162,123$ form a hexagon with 
center 1 . The substitution $S_{1}$ leaves 1 invariant and permutes the sides of the hexagon cyclically: $156,162,123,134,147,175$. The substitution $T$ changes the hexagon into the hexagon with center (5): $517,572,524,543$, 536,561 . The mapping process of the polyhedron of 14 triangles upon a torus is also verified by the formula

$$
k=V+F-S-7+14-21=0 .
$$

Moreover $2-k=2=2 p, p=1$.

The hexagon with 1 as a center has the cycle $U=\left(\begin{array}{ll}347562 & 4\end{array}\right)$ of order 6, which interchanges the sets of triple systems as follows:

$$
\begin{aligned}
& 1113344 \quad 1113347 \\
& 3767672 \quad 3464762 \\
& 4522565 \quad 2755625 \text {. }
\end{aligned}
$$

Now the powers of $U$ are

$$
\begin{aligned}
& U=(347562), \quad U^{2}=(376)(452), \quad U^{3}=(35)(46)(72), \\
& U^{4}=(367)(425), \quad U^{5}=(326574), \quad U^{6}=1 \text {. }
\end{aligned}
$$

Thus the entire group leaving the polyhedron, and in particular the polyhedron of 7 hexagons, on the torus invariant is

$$
\left(S_{1}, S_{2}, \cdots, S_{7}\right)(1, T)\left(1, U, U^{2}\right) \equiv G_{42},
$$

as is well known.*

4. Triple systems of order 9 with double couples. The triple system obtained from

$$
\left\|\begin{array}{lll}
1 & 2 & 3 \\
4 & 5 & 6 \\
7 & 8 & 9
\end{array}\right\| \equiv \begin{array}{llllllllllll}
1 & 1 & 1 & 1 & 2 & 2 & 2 & 3 & 3 & 3 & 4 & 7 \\
2 & 4 & 5 & 6 & 4 & 6 & 5 & 5 & 4 & 6 & 5 & 8 \\
3 & 7 & 9 & 8 & 9 & 7 & 8 & 7 & 8 & 9 & 6 & 9
\end{array}
$$

has a group which is doubly transitive, since 1, 2 may be replaced by any two other letters, say 5 and 8:

$$
\left\|\begin{array}{lll}
5 & 8 & 2 \\
1 & 4 & 7 \\
9 & 3 & 6
\end{array}\right\|
$$

Or we have the substitution $(154)(283)(796)$ which leaves the triple

* Brahana, Regular maps on an anchor ring, American Journal of Mathematics, vol. 48 (1926), pp. 225-240. 
system invariant. Moreover the substitutions which leave 1, 2 invariant also leave 3 invariant and form a group of order 6:

1, (47)(58)(69), (456)(798), (465)(789), (59)(67)(48), (68)(49)(57).

Hence the group which leaves the triple system invariant is a $G_{432}$ of order $9 \cdot 8 \cdot 6=432$, since a group on $n$ elements which is $k$-fold transitive has the order equal to the product of $n(n-1) \cdots(n-k+1)$ and the order of the group which leaves $k$ of the elements fixed. The $G_{432}$ is also the holomorph of the non-cyclic abelian group $\Gamma_{9}$ of order 9:

in which

$$
\Gamma_{9}\left(S_{1}, S_{2}, S_{3}, S_{4}\right) \text {, }
$$

$$
\begin{aligned}
& S_{1}=123 \cdot 456 \cdot 789, \\
& S_{2}=147 \cdot 258 \cdot 369, \\
& S_{3}=159 \cdot 267 \cdot 348, \\
& S_{4}=168 \cdot 249 \cdot 357 .
\end{aligned}
$$

Two triple systems $\Delta_{9}{ }^{3}$ without a common triple form a closed surface with three double vertices:

$$
\begin{aligned}
& \left\|\begin{array}{lll}
1 & 2 & 3 \\
4 & 5 & 6 \\
7 & 8 & 9
\end{array}\right\|=\begin{array}{llllllllllll}
1 & 1 & 1 & 1 & 2 & 2 & 2 & 3 & 3 & 3 & 4 & 7 \\
2 & 4 & 5 & 6 & 4 & 5 & 6 & 4 & 5 & 6 & 5 & 8 \\
3 & 7 & 9 & 8 & 9 & 8 & 7 & 8 & 7 & 9 & 6 & 9
\end{array} \\
& \left\|\begin{array}{lll}
1 & 2 & 4 \\
3 & 5 & 9 \\
7 & 6 & 8
\end{array}\right\|=\begin{array}{llllllllllll}
1 & 1 & 1 & 1 & 2 & 2 & 2 & 3 & 3 & 4 & 4 & 7 \\
2 & 3 & 5 & 6 & 3 & 5 & 7 & 4 & 5 & 5 & 8 & 6 \\
4 & 7 & 8 & 9 & 8 & 6 & 9 & 6 & 9 & 7 & 9 & 8 .
\end{array}
\end{aligned}
$$

The surface can be disected and mapped on a rectangle, Fig. 2, then, as in Fig. $1 b$, on a cylinder, and finally on a torus.

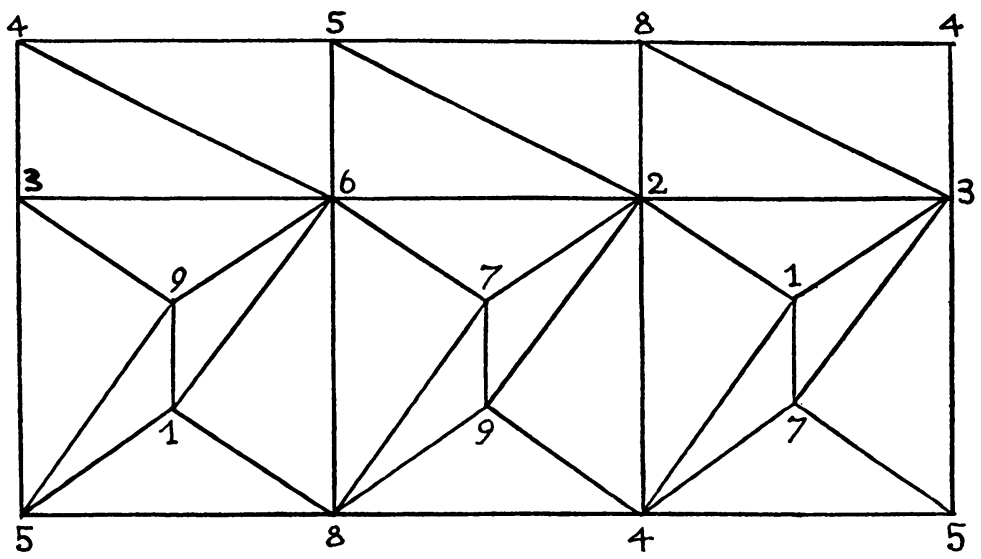

Frg. 2 
There is to be noticed this peculiarity in the polyhedral configuration for $n=9$, that the three vertices $1,7,9$ are double vertices, each with two distinct cycles of triangles. By Euler's formula

$$
k=V-S+F=(6+3 \cdot 2)-36+24=0,
$$

so that $2-k=2$ is the order of connectivity.

From the rectangular figure it is apparent that the cylindrical configuration, with the edges $\overline{534}$ joined, is left invariant by the cyclic substitutions (rotations around axis of cylinder)

moreover by

$$
(458)(236)(197)=T,
$$

$$
\begin{aligned}
& S_{1}=(28)(35)(46)(79), \\
& S_{2}=(17)(25)(34)(68), \\
& S_{3}=(19)(38)(56)(24) .
\end{aligned}
$$

Now

$$
\begin{aligned}
& S_{1} S_{3}=T=(236)(458)(719), \\
& S_{1} S_{2}=(263)(485)(917)=T^{2}, \\
& S_{2} S_{3}=(791)(263)(485)=T^{2} .
\end{aligned}
$$

Hence there is a non-cyclic group $G_{6}$ of order $6\left(1, T, T^{2}, S_{1}, S_{2}, S_{3}\right)$ which leaves the closed surface invariant.

5. Triple systems of even order with double couples. The simplest case of this sort is for $n=6 \mu, \mu=1$, or $n=6$, with $x=2 \mu(6 \mu-1)=10$.

Such a system is for example

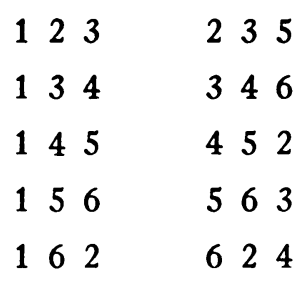

which form a closed surface as shown when mapped on a plane, Fig. 3. It is unilateral and can be mapped on a projective plane. The formula gives

$$
V-S+F=10+6-15=1=k
$$

or $2-k=1$ for the order of connectivity. The substitutions which leave the surface in its composition of triples invariant are the cycles of order 3 which rotate Fig. 3 about its center. Such a cycle is $123 \cdot 465,132 \cdot 456$. But every triple may be chosen at the center, thus we have $10 \cdot 2$ such substitutions. 
Then there are cycles of order 5 which leave one point invariant, for example 1, i.e., (23456), (24635), (25364), (26543), or $6.4=24$ such substitutions. Finally there are 15 substitutions of order 2, each forming a spear head like (13) (24), or (16) (34), (16) (25). Hence there are altogether $1+15+20+24$ $=60$ substitutions which leave the triple system invariant. If we join the centers of the five triangles around each vertex we obtain the unilateral surface mapped into 6 pentagons, i.e., a unilateral polyhedrnn of 6 pentagons on the projective plane and 10 vertices is left invariant by the icosahedral group.

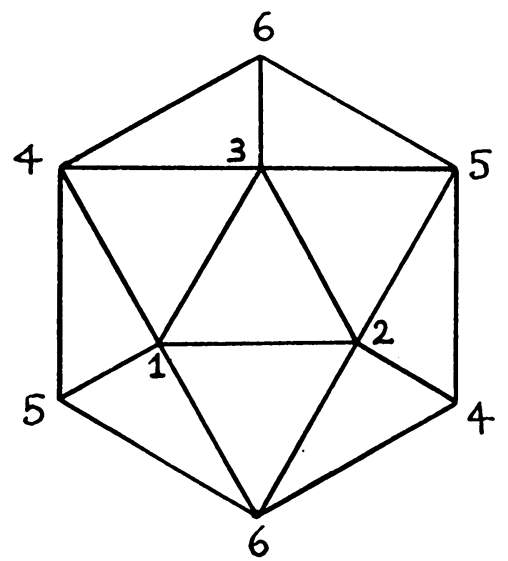

Frg. 3

Considering the remaining 10 triples, they form also a triple system with double couples

$\begin{array}{lllllll}1 & 2 & 4 & & 2 & 4 & 3 \\ 1 & 4 & 6 & & 4 & 6 & 5 \\ 1 & 6 & 3 & & 6 & 3 & 2, \\ 1 & 3 & 5 & & 3 & 5 & 4 \\ 1 & 5 & 2 & & 5 & 2 & 6\end{array}$

which is left invariant by the same icosahedral group. As can easily be verified, each triple is common to six distinct triple systems. With every triple system is associated the complementary triple system formed by the other 10 of the total of 20 triples. In a triple system and its complement all 20 triples are contained. Hence there are altogether 12 distinct triple systems and in this complex each triple is contained just 6 times. 


\section{Multiple Systems}

1. Systems $\Delta_{n}^{k}$. $n$ elements $1,2,3, \cdots, n$ form $\left(\begin{array}{l}n \\ k\end{array}\right) k$-tuples and $\left(\begin{array}{c}n \\ k-1\end{array}\right)$ $(k-1)$-tuples. It is proposed to form a system $\Delta_{n}^{k}$ of $k$-tuples with the property that it contains each $(k-1)$-tuple just once.

Let $x$ be this number; then since every $k$-tuple contains $k(k-1)$-tuples

$$
k x=\left(\begin{array}{c}
n \\
k-1
\end{array}\right), \text { or } x=\frac{n(n-1)(n-2) \cdots(n-k+2)}{1 \cdot 2 \cdot 3 \cdots(k-1) k},
$$

which must be an integer. The condition that this be an integer is however not sufficient. Let $y$ be the number of $k$-tuples of $\Delta_{n}^{k}$ containing the letter 1 . Then all $(k-1)$-tuples of $\Delta_{n}^{k}$ containing the letter 1 are in these $k$-tuples. A $(k-2)$-tuple formed from the remaining $n-1$ letters cannot appear in more than one of the $y k$-tuples. On the other hand each of these $(k-2)$ tuples is contained in the $y k$-tuples. Thus in each of the $y k$-tuples, there are $\left(\begin{array}{c}k-1 \\ k-2\end{array}\right)(k-2)$-tuples. Hence

$$
y\left(\begin{array}{l}
k-1 \\
k-2
\end{array}\right)=\left(\begin{array}{l}
n-1 \\
k-2
\end{array}\right)
$$

or

$$
y=\frac{(n-1)(n-2) \cdots(n-k+2)}{(k-1)(k-2) \cdots 2 \cdot 1}
$$

is the number of $k$-tuples of $\Delta_{n}^{k}$ with a given letter just once in each of the $y k$-tuples. For a $\triangle_{n}^{k}$ system $y$ must be an integer, which is another necessary condition. Next we might find the number of $k$-tuples that each contain a given couple $i k$ and so forth.

2. $\triangle_{n}^{4}$. To illustrate this, take $k=4$; then

$$
x=\frac{n(n-1)(n-2)}{1 \cdot 2 \cdot 3 \cdot 4}, \quad y=\frac{(n-1)(n-2)}{3 \cdot 2} .
$$

In this case $n-2$ elements which form quadruples with $i k$ must form $\frac{1}{2}(n-2)$ couples which with $i k$ form all possible distinct triples each containing $i k$. From this follows that $n$ must be even. Thus $n=2 m$, and $y=\frac{1}{3}(m-1)$ $(2 m-1)$, and either $m=3 \mu+1$, or $m=\frac{1}{2}(3 \mu+1)$. In the latter case $\mu=2 \gamma+1$ and $m=3 \gamma+2$.

For quadruple systems of order $n$ we have therefore either $n=6 \mu+2$, or $n=6 \mu+4$, and the number of quadruples in $\triangle_{n}^{k}$ is accordingly

$$
x=\frac{1}{2}(3 \mu+1)(6 \mu+1) \mu, \quad \text { or } \quad x=\frac{1}{2}(3 \mu+2)(2 \mu+1)(3 \mu+1) .
$$

In such a system each element occurs $\mu(6 \mu+1)$ or $(2 \mu+1)(3 \mu+1)$ times. 
3. Quadruple systems of lowest order $\Delta_{8}{ }^{4}$. Next to the quadruple system on four elements which consists of one quadruple 1234, containing four triples $123,124,134,234$, and which is left invariant by the symmetric group on four elements, the quadruple systems of lowest order can be formed with 8 elements, such that each system contains 14 quadruples and each possible triple once, and every couple three times, and each letter 7 times.

It is not difficult to devise a graphical scheme to establish such quadruple systems. One is to number the vertices of a cube from 1 to 8 in any order. Then the quadruples of the system consist first of 6 faces, second of the 6 rectangles each determined by two opposite edges, third by the two tetrahedrons inscribed in the cube:

\section{$\begin{array}{llllllllllllll}1 & 4 & 1 & 2 & 1 & 3 & 1 & 3 & 2 & 1 & 1 & 2 & 2 & 1\end{array}$ \\ $26 \begin{array}{llllllllllll}2 & 6 & 3 & 2 & 4 & 2 & 5 & 3 & 4 & 3 & 5 & 4\end{array}$ \\ 376465546654757 \\ $\begin{array}{llllllllllllll}5 & 8 & 7 & 8 & 8 & 7 & 7 & 8 & 78 & 8 & 8 & 68 & 8\end{array}$.}

The problem now is to determine the order of the group of degree 8 which will permute the quadruples of the system, in which a quadruple is defined as a set of four numbers irrespective of the order in which they are written. In the first place it is evident that the octahedral group written on 8 letters leaves the system invariant and hence is a subgroup of the group in question. Moreover, as any three letters on the cube can be replaced by any three other letters and the remaining one so distributed over the remaining vertices as to give rise to the same quadruple systems, the group will be triply transitive. The substitutions of the group which leave 3 letters unchanged, for instance 123, also must leave 5 unchanged. These are 1, (46)(78), (47) (68), (48) (67), which form a subgroup of order 4. Hence according to a well known theorem that the order of a group of degree $n$ which is $k$-fold transitive is equal to the product of $n(n-1) \cdots(n-k+1)$ and the order of the group which leaves $k$ elements unchanged, the order of the group of the quadruple system is

$$
8 \cdot 7 \cdot 6 \cdot 4=1344
$$

This order may also be obtained by establishing the number of distinct quadruple systems. As every triple must be in a system,

$$
1234, \quad 1235, \quad 1236,1237,1238
$$

must belong to different quadruple systems. The problem then is to find how many quadruple systems can be formed with each of those quadruples fixed. Considering 1235, for example, we can form 24 permutations with 
4678, each of which with 1235 defines a quadruple system. But any two successive transpositions of different letters, like (47)(68), leaves the quadruple system invariant. As there are 4 such double transpositions, there are 6 distinct substitutions, (48), (67), (47), (68), (78), (46), which will give three distinct quadruple systems. Thus either (46), (47), (48), or (78), (68), (67), give three distinct systems; likewise the product of any two, i.e., (46)(47), (47)(48), (48)(46), three more, i.e. altogether 6 quadruple systems associated with 1235. Thus 4876 is obtained by the substitution (78) (67) or (47) (68) (46) (47) from 4768; likewise 4687 from 4768 by (67) (87) or (678). As there are altogether 8 ! substitutions and 30 distinct quadruple systems, each quadruple must be invariant in $8 !: 30=1344$ substitutions, which form the above $G_{1344}$.

As to the structure of this group it contains a $G_{8}$ :

a $G_{8}^{\prime}$ :

$(12)(35)(47)(68)$

$(13)(25)(46)(78)$

$(15)(23)(48)(67)$

$(18)(26)(37)(45)$

$(16)(28)(34)(57)$

$(17)(24)(38)(56)$

$(14)(27)(36)(58)$;

$(1253)(48)$

(15)(23)

(1352)(48)

(12)(35)

(23) (48)

(13)(25)

(15)(48) ;

a $G_{7}$ :

(1234587) cyc. ;

a $G_{3}$ :

$(235)(478)$ cyc. ;

moreover the subgroups

$$
\begin{aligned}
G_{64} & =G_{3} \cdot G_{8}^{\prime}, \quad G_{8} \cdot G_{7}=G_{56}, \quad G_{8} \cdot G_{3}=G_{24}, \\
G_{7} \cdot G_{3} & =G_{21}, \quad G_{8} \cdot G_{7} \cdot G_{3}=G_{168} \quad \text { (not simple). }
\end{aligned}
$$


4. Configuration of two quadruple systems without a common quadruple. If we interpret the eight elements as eight points in space of four dimensions, every quadruple will be represented by a tetrahedron. Two quadruple systems without a common quadruple can be joined so that each face (triple) of the fourteen tetrahedrons in one system will coincide with the same triple-face of the tetrahedron in the second system in which it occurs. In this manner we obtain a closed hypersurface $P_{23}$ in $S_{4}$ bounded by 28 tetrahedral cells.

If in an $S_{n}$ there are, for a polyhedron, $r_{0}$ vertices, $r_{1}$ edges, $r_{2}$ faces, $r_{3}$ cells, $r_{1}$ four-spaces bounding, etc., then for an Eulerian polyhedron

$$
1-r_{0}+r_{1}-r_{2}+r_{3}+\cdots+(-1)^{n-1} r_{n-1}=0 \text {. }
$$

Here $r_{n-1}$, the number of $(n-1)$-spaces bounding the polyhedron, is 1 . Now for our 28-cell in $S_{4}$ we have

$$
1-8+28-56+28-1=-8 \text {. }
$$

Hence the 28-cell in $S_{4}$ does not belong to the ordinary polytope.

The cells of a $P_{28}$ may be represented for example by

$$
\begin{gathered}
Q_{14}\left\{\begin{array}{llllllllllllll}
1 & 1 & 1 & 1 & 1 & 1 & 1 & 2 & 2 & 2 & 2 & 3 & 3 & 4 \\
2 & 2 & 2 & 3 & 3 & 4 & 5 & 3 & 3 & 4 & 5 & 4 & 5 & 6 \\
3 & 4 & 6 & 4 & 7 & 5 & 6 & 4 & 6 & 5 & 7 & 5 & 6 & 7 \\
5 & 7 & 8 & 6 & 8 & 8 & 7 & 8 & 7 & 6 & 8 & 7 & 8 & 8
\end{array},\right. \\
Q_{14}^{\prime}\left\{\begin{array}{llllllllllllll}
1 & 1 & 1 & 1 & 1 & 1 & 1 & 3 & 4 & 2 & 2 & 2 & 2 & 3 \\
2 & 3 & 5 & 3 & 4 & 2 & 2 & 4 & 5 & 3 & 5 & 3 & 4 & 6 \\
4 & 5 & 6 & 4 & 6 & 3 & 7 & 5 & 7 & 5 & 6 & 4 & 6 & 7 \\
5 & 7 & 8 & 8 & 7 & 6 & 8 & 6 & 8 & 8 & 7 & 7 & 8 & 8
\end{array},\right.
\end{gathered},
$$

so that

$$
P_{28}=Q_{14}+Q_{14}^{\prime}
$$

in which $Q_{14}$ and $Q_{14}^{\prime}$ are two quadruple systems without a common quadruple. The problem is to find the group of degree 8 which will leave $P_{28}$ invariant. There are substitutions which leave any two elements in $Q_{14}$ and the same two elements in $Q_{14}^{\prime}$ fixed and permute $Q_{14}$ and $Q_{14}^{\prime}$; for example, $S=(25)$ (34) (18) which leaves (1) and (7), $T=(37)$ (46) (58) which leaves (1) and (2), $U=(13)$ (68) (27) which leaves (4) and (5) invariant and also permutes $Q_{14}$ and $Q_{14}^{\prime}$. The product of any such two substitutions is a substitution which leaves each $Q_{14}$ and $Q_{14}^{\prime}$ invariant, for example

$$
S T=(2847365), \quad S U=(134)(257) \text {. }
$$


Thus for each element there is a cyle of order 7 which leaves this element and each $Q_{14}$ and $Q_{14}^{\prime}$ invariant. It is seen that the substitutions of the group which leave $P_{28}$ invariant, are of two kinds: those that permute $Q_{14}$ and $Q_{14}{ }^{\prime}$ and those that leave both $Q_{14}$ and $Q_{14}^{\prime}$ invariant. Another substitution which leaves (1) and (2) fixed and permutes $Q_{14}$ and $Q_{14}^{\prime}$ is $W=(368745)$, for which $W^{2}=(384)(675), W^{3}=(37)(46)(58)=T . S U T=(17285364)$ is a substitution of order 8 which permutes $Q_{14}$ and $Q_{14}^{\prime} .(S U T)^{2}=(1256)(3478)$ and $(S U T)^{4}$ $=(15)$ (26) (37) (48) are substitutions which transform $Q_{14}$ and $Q_{14}^{\prime}$ into themselves. Hence there are operators of order 2, 3, 4, 7 which transform $Q_{14}$ and $Q_{14}^{\prime}$ into themselves, and operators of order 2, 6, 8 which permute $Q_{14}$ and $Q_{14}^{\prime}$.

The group is again triply transitive and the substitutions which leave three elements, say (1), (2), (3), invariant cannot also leave (5) and (6) invariant (outside of identity) hence 5 and 6 must be permuted, likewise 7 and 8 , but these assumptions lead to contradictions, so that the only substitution which leaves $1,2,3$, in $Q_{14}+Q_{14}^{\prime}$, invariant is identity. Hence the group leaving $P_{28}$ invariant is of order $8 \cdot 7 \cdot 6 \cdot 1=336$. The odd substitutions of this group permute $Q_{14}$ and $Q_{14}^{\prime}$ while the even substitutions form the simple $G_{168}$ which leaves both $Q_{14}$ and $Q_{14}^{\prime}$ invariant. There is

$$
G_{336}=\left(1, C, C^{2}, \cdots\right)\left(1, A, A^{2}, \therefore\right)\left(1, B, B^{2}, \cdots\right),
$$

where

$$
\begin{aligned}
& A=(285743), \\
& B=(1234567), \\
& C=(17285364) .
\end{aligned}
$$

The orders of $A$ and $C$ are 6 and 8 respectively. The product $C^{\alpha} A^{\beta}$ leaves $Q_{14}$ and $Q_{14}^{\prime}$ invariant, when $\alpha+\beta=2 m$. As $A^{6}=1, C^{8}=1$, we may solve this equation for $\alpha+\beta \leqq 12, \alpha \leqq 8, \beta \leqq 6$, and find easily 24 solutions, including identity.

Hence there are 24 substitutions of the form $C^{\alpha} A^{\beta}$ which leave $\Delta_{4}^{8}$ and $\Delta_{4}^{\prime 8}$ invariant, consequently leave the configuration $\Delta_{4}^{8} \cdot \Delta_{4}^{8}$, or the polyhedron $P_{28}$ in $S_{4}$ invariant.

That configurations admitting finite groups are not limited to the types $\Delta_{n}^{k}$ will appear from the following example.

5. A certain quadruple system on 16 elements. It is proposed to construct a quadruple system on 16 elements $1,2, \cdots, 16$ such that every element occurs, just 6 times, i.e., in six quadruples of the system. 
Let $x$ be the number of quadruples; then there are $4 x$ elements in the system. But as every element occurs in six quadruples, it follows that

$$
\frac{4 x}{6}=16 \text {, }
$$

and $x=24$. Thus such a system must have 24 quadruples. Such a system is for example

$\begin{array}{rrrr}1.2 \cdot 3 \cdot 4 & 1 \cdot 5 \cdot 4 \cdot 8 & 5 \cdot 9 \cdot 12 \cdot 8 & 1 \cdot 4 \cdot 16 \cdot 13 \\ 5 \cdot 6 \cdot 7 \cdot 8 & 2 \cdot 6 \cdot 7 \cdot 3 & 9 \cdot 13 \cdot 16 \cdot 12 & 1 \cdot 2 \cdot 14 \cdot 13 \\ 9 \cdot 10 \cdot 11 \cdot 12 & 8 \cdot 12 \cdot 11 \cdot 7 & 10 \cdot 14 \cdot 15 \cdot 11 & 1 \cdot 5 \cdot 9 \cdot 13 \\ 13 \cdot 14 \cdot 15 \cdot 16 & 12 \cdot 16 \cdot 15 \cdot 11 & 6 \cdot 10 \cdot 11 \cdot 7 & 2 \cdot 3 \cdot 15 \cdot 14 \\ 1.5 \cdot 6 \cdot 2 & 5 \cdot 9 \cdot 10 \cdot 6 & 7 \cdot 11 \cdot 15 \cdot 3 & 6 \cdot 10 \cdot 14 \cdot 2 \\ 8 \cdot 7 \cdot 3 \cdot 4 & 9 \cdot 13 \cdot 14 \cdot 10 & 3 \cdot 16 \cdot 15 \cdot 4 & 8 \cdot 4 \cdot 16 \cdot 12 .\end{array}$

There are exactly 32 couples each of which occurs exactly 3 times in the system. The 24 quadruples may be grouped into 8 octuples:

$\begin{array}{rrrr}1.2 \cdot 3 \cdot 4 & 5 \cdot 6 \cdot 7 \cdot 8 & 9 \cdot 10 \cdot 11 \cdot 12 & 13 \cdot 14 \cdot 15 \cdot 16 \\ 5 \cdot 6 \cdot 7 \cdot 8 & 9 \cdot 10 \cdot 11 \cdot 12 & 13 \cdot 14 \cdot 15 \cdot 16 & 1 \cdot 2 \cdot 3 \cdot 4 \\ 8 \cdot 4 \cdot 1 \cdot 5 & 5 \cdot 6 \cdot 2 \cdot 1 & 6 \cdot 10 \cdot 14 \cdot 2 & 8 \cdot 7 \cdot 3 \cdot 4 \\ 12 \cdot 16 \cdot 13.9 & 10 \cdot 14 \cdot 13 \cdot 9 & 7 \cdot 11 \cdot 15 \cdot 3 & 12 \cdot 11 \cdot 15 \cdot 16,\end{array}$

so that every octuple contains exactly 6 of the quadruples of the system. But the 48 quadruples thus obtained are two by two equal, so that there are only the 24 original distinct quadruples. Now between the number of elements, couples, quadruples and octuples exists the identity

$$
1-16+32-24+8-1=0
$$

which is Euler's formula for the 8-cell, or cube in four dimensions. Geometrically this follows from the following consideration. The 16 elements may be represented by 16 generic points in euclidean four-space. By a continuous deformation of space, more precisely by a transformation of at least the second order

$$
\rho x_{i}^{\prime}=\frac{\sum_{k, l=1}^{4} a_{i k l} x_{k} x_{l}+d_{i k} x_{k}+b_{i}}{\sum_{k, l=1}^{4} a_{5 k l} x_{k} x_{l}+C_{i k} x_{k}+d} \quad(i=1,2,3,4),
$$


depending on 74 effective constants, 16 generic points, determined by $4 \cdot 16=64$ constants, may always be transformed into the 16 vertices of an 8-cell. Thus when they are transformed in the order as indicated by the above quadruple system, the elements, couples, quadruples, and octuples of the system form the vertices, edges, faces and cubes of an 8-cell in four dimensions. The octahedral group attached to each cube of the 8-cell leaves this cube invariant and induces a definite transformation of the remaining cubes among themselves, thus transforming the whole 8-cell into itself. With each cube there is associated such an octahedral group. Hence there are $8 \cdot 24=192$ movements of the 8-cell into itself. Or the group associated with the quadruple system is a $G_{192}$, which is well known as the polyhedral group which leaves the 8-cell invariant.

Every permutation of the 16 elements marked off in quadruples as in

$$
\begin{array}{llllllllllllll}
1 & 2 & 3 & 4 & 5 & 6 & 7 & 8.9 & 10 & 11 & 12 \cdot 13 & 14 & 15 & 16
\end{array}
$$

and formed into a quadruple system as induced by this grouping gives rise to a quadruple system which is invariant in a $G_{192}$.

6. Quintuple and higher systems. By a similar procedure, as in $\Delta_{n}{ }^{4}$ it is found that the lowest possible non-trivial quintuple system is a $\Delta_{11}{ }^{5}$ with 66 quintuples. In such a system every letter occurs 30 times, every couple 12 times, every triple 4 times, every quadruple once.

Professor Carmichael shows that the group which leaves this system invariant is a four-fold transitive Mathieu group of degree 11 and order $11 \cdot 10 \cdot 9 \cdot 8$.

The existence proofs for quintuple systems for $n>11$, and in general of $k$-tuple systems, seem to be difficult, likewise the solution of the problem of complete sets of $k$-tuple systems for possible values of $n$. In any case these problems are closely connected with group theory.

As in the case of the 8-cell in $S_{4}$, other conditions than the existence of each $(k-1)$-tuple in a set of $k$-tuples may be imposed on $n$ elements. We get accordingly different configurations and groups in which they are invariant.

UNTVERSITY OP ILLINOIS, URBANA, ILL. 